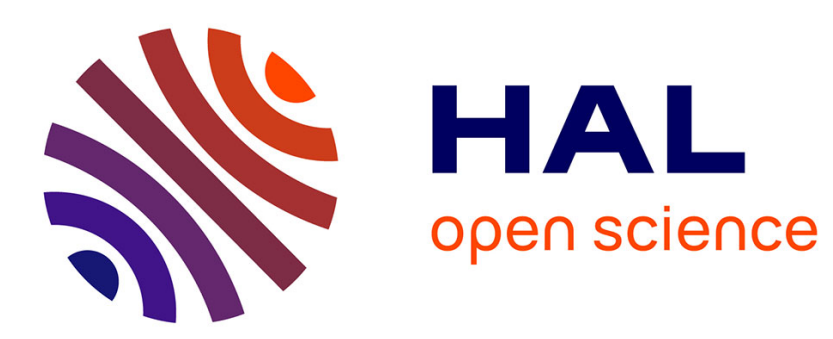

\title{
Controlling the buckling instability of drying droplets of suspensions through colloidal interactions
}

É. Lintingre, G. Ducouret, F. Lequeux, L. Olanier, T. Périé, L. Talini

\section{To cite this version:}

É. Lintingre, G. Ducouret, F. Lequeux, L. Olanier, T. Périé, et al.. Controlling the buckling instability of drying droplets of suspensions through colloidal interactions. Soft Matter, 2015, 11 (18), pp.36603665. 10.1039/C5SM00283D . hal-01141980

\section{HAL Id: hal-01141980 \\ https://hal.sorbonne-universite.fr/hal-01141980}

Submitted on 14 Apr 2015

HAL is a multi-disciplinary open access archive for the deposit and dissemination of scientific research documents, whether they are published or not. The documents may come from teaching and research institutions in France or abroad, or from public or private research centers.
L'archive ouverte pluridisciplinaire HAL, est destinée au dépôt et à la diffusion de documents scientifiques de niveau recherche, publiés ou non, émanant des établissements d'enseignement et de recherche français ou étrangers, des laboratoires publics ou privés. 


\title{
Controlling the buckling instability of drying droplet of suspensions through colloidal interactions
}

\author{
É. Lintingre, ${ }^{a, b}$ G.Ducouret, ${ }^{a, b}$ F. Lequeux, ${ }^{a, b}$ L. Olanier, ${ }^{a, b}$ T. Périé, ${ }^{c}$ and L. Talini, ${ }^{a, b}$
}

The present study focuses on the drying of droplets of colloidal suspensions using Leidenfrost effect. At the end of drying, grains show different morphologies: cup or spheres depending on the ionic strength or zeta potential of the initial suspension. High ionic strengths and low absolute zeta potential values lead to spherical morphologies. A modelisation based on the calculations of DLVO potentials has been implemented to extract a critical pressure which provides a quantitative criterion for buckling whatever the initial formulation. Particularly, the buckling time is quantitatively predicted from the interparticular interactions and shows an excellent agreement with experimental values.

\section{$1 \quad$ Introduction}

The drying of colloidal suspensions has been extensively studied in the past years ${ }^{1-5}$. The observed phenomena, such as buckling and fracture, are of fundamental interest but also of importance in many industrial processes, in pharmaceutics $^{6}$, ceramics ${ }^{7,8}$, food processing ${ }^{9}$, coatings ${ }^{10}$ or porous materials engineering ${ }^{11}$. It is now well understood that one key parameter is the Péclet number that compares the diffusion time of the colloidal particle over a characteristic distance with the drying time:

$$
P e=\frac{\tau_{d i f f}}{\tau_{d r y}}
$$

For large Péclet numbers, i.e. fast drying conditions, the particles are transported to the interface with air and concentrate since diffusion is too slow to prevent building of concentrations gradients. A concentrated layer then forms, which can further become solid and buckle. Owing to the small values of the diffusion coefficient of colloidal particles, the condition Pe >> 1 is met even for relatively slow drying processes; for instance drying within one hour of a millimetre-sized sample corresponds to a Péclet number larger than 100 for a coefficient diffusion of $10^{-12} \mathrm{~m}^{2} \cdot \mathrm{s}^{-1}$.

The case of the fast drying of droplets of colloidal suspensions has been particularly investigated owing to the richness of the different morphologies of the dried grains: from perfect spheres to buckled shapes such as deflated balloons or doughnuts ${ }^{12-17}$. From a practical point of view, there is a need to understand the drying mechanisms involved during spray drying, a widely used process that consists in drying aerosols for manufacturing large amounts of fine powders. In particular, the key parameters that control the final shape of the dried droplets have not been entirely elucidated.

A physical picture was first suggested by Tsapis et al. ${ }^{12}$ who observed the buckling of drying droplets of suspensions of charged colloids. As the droplet dries, the solvent flux toward the droplet's surface results in the formation of a shell concentrated in particles. Although the interaction between colloids is repulsive, they can nonetheless aggregate when the Darcy pressure resulting from the solvent flow overcomes the net colloidal repulsive stress. A solid viscoelastic shell thus forms, which can further buckle as drying proceeds and the droplet's volume decreases. Consistently with that picture, Tsapis et al. observed that an addition of salt in the colloidal suspension decreases the time at which the drop buckles, since it decreases the net repulsive force between colloidal particles. Therefore, no spherical droplet was ever obtained after drying of the stable (from a colloidal point of view) suspensions.

In the present paper, we study the drying of droplets of zirconia colloidal suspensions according to the colloidal interactions between particles. We have tuned the Debye length and/or the zeta potential of the colloidal particles in order to vary the interaction potential from a repulsive to an attractive potential. We observed the different morphologies after drying (figures 1 and 2). When the potential is repulsive enough to prevent particle aggregation, i.e. when the energy barrier is large

\footnotetext{
${ }^{a}$ École Supérieure de Physique et de Chimie Industrielles de la Ville de Paris ParisTech, PSL Research University, Sciences et Ingénierie de la matière Molle, CNRS UMR7615, 10, Rue Vauquelin, F-75231 Paris Cedex 05. France

${ }^{\mathrm{b}}$ Sorbonne-Universités, UPMC Univ. Paris 06, SIMM, 10, Rue Vauquelin, F-75231 Paris Cedex 05, France

${ }^{c}$ Saint-Gobain CREE, 550 avenue Alphonse Jauffret, BP 20224, 84306 Cavaillon - France
} 


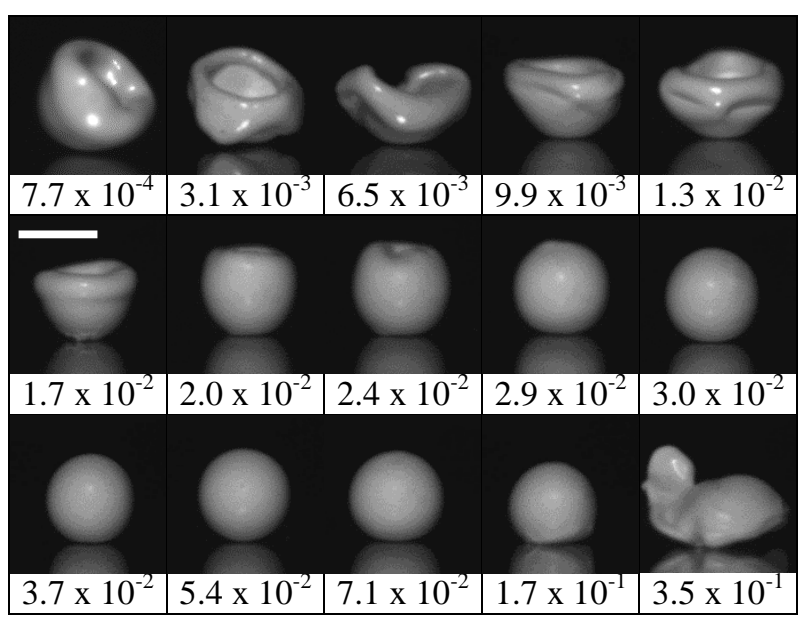

Fig. 1: Photographs of grains obtained from the drying of droplets of zirconia aqueous suspensions with different ionic strength (val ues in mol/L). Scale bar represents $1 \mathrm{~mm}$.

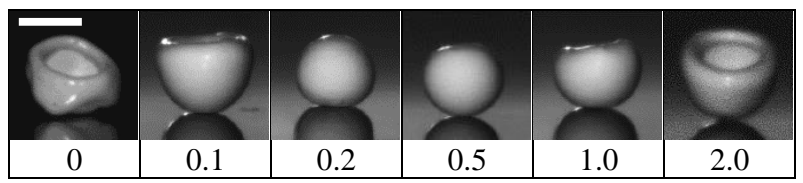

Fig. 2: Photographs of grains obtained from the drying of droplets of zirconia aqueous suspensions with different concentrations of $P A A$ (values in $\%$ wt/zirconia mass). Scale bar represents $1 \mathrm{~mm}$.

enough, we find results in agreement with the ones of Tsapis. In addition, we show that, when the energy barrier is further lowered and particles form aggregates, buckling of the droplet is delayed. We explain that observation and provide a quantitative description of the results. Finally, we demonstrate that for very small energy barriers the droplets remain spherical during drying.

We relate that result to the formation of fractal aggregates. Therefore, we provide for the first time the key parameters to fully control the final shape of dried droplets of colloidal suspensions.

\section{Experimental details}

Drying of droplets levitating above a hot plate is performed thanks to Leidenfrost effect. When the plate is hot enough, the droplet does not boil but levitates on its own vapour, acting as an insulating shield as long as there is enough solvent to feed it $^{18}$.

A polished brass concave plate is placed on an adjustable heating surface (from $20^{\circ} \mathrm{C}$ to $400^{\circ} \mathrm{C}$ ). Keeping the droplet at a fixed position during drying is given by the concave geometry and the quasi-frictionless motion of levitation. Polishing was performed with diamonds pastes with decreasing granulometries to obtain a mirror effect and lower the critical Leidenfrost temperature. A spin-coating of the concave surface with a nanosuspension of functionalized hydrophobic silica (GLACO) optimizes aqueous droplet levitation. The initial volume of the droplet is fixed to $2 \mu \mathrm{L}$ to obtain droplets with diameters smaller than the capillary length. Consequently, liquid levitated droplets were always spherical at the beginning of drying and buckling or deformation phenomenon cannot be explained by gravity. During drying, volume decreases as the water evaporates and feeds the vapour under the droplet. Drying ends when Leidenfrost effect stops: the levitating droplet has turned into a motionless grain.

The suspensions are colloidal zirconia particles suspended in water. Zirconia powder (provided by Saint-Gobain ZirPro) is de-agglomerated in water and milled with a repetable water milling step to reach a median radius of $110 \pm 30 \mathrm{~nm}$. Particle size distribution is measured with Horiba laser scattering particle size distribution analyser LA-950 after water-milling step. In all the samples, the zirconia volume fraction was kept at $15.5 \%$ (corresponding to $50 \% \mathrm{wt}$ ).

The different formulations were prepared with the additives reported in Table 1 and homogenized during 24 hrs. 
Table 1: The different types of additives used throughout the experiments.

\begin{tabular}{ccc}
\hline Matter & Supplier & Comments \\
\hline $\mathrm{NaCl}$ & Sigma Aldrich & $\geq 99 \%$ \\
Polyacrylic acid PAA & Polysciences Inc. & $\mathrm{M}_{\mathrm{w}}=50,000 \mathrm{~g} / \mathrm{mol}$
\end{tabular}

In pure water, $\mathrm{pH}$ is equal to 5.5 after dissolution of zirconia particles. They bear positive charges owing to the ionization of hydroxyl groups at their surfaces. Zeta potential measurements were performed with a zetameter Field ESA from CAD Instruments. Addition of $\mathrm{NaCl}$ results in an increase of ionic strength and thus an increased screening of charges at constant zeta potential.

The zeta potential can be varied by adding species such as polyacrylic acid (PAA) that adsorbs onto the particle surfaces. PAA bearing negative charges, the resulting net charge of the particles can thus be decreased and even become negative for large addition of polymer. Zeta potential varies from $30 \mathrm{mV}$ to $-30 \mathrm{mV}$ with PAA addition.

Since the dispersed suspensions are in a metastable state, special care has been taken to perform experiments at a fixed time after their preparation. 24 hours after their preparation, a small volume of suspension is taken up with a micropipette and a $2 \mu \mathrm{L}$ droplet is deposited on the hot plate. A 1960x1420 camera captures side views of the drying droplet at $5 \mathrm{~Hz}$ frequency from which drop radius as a function of time can be measured. The typical drying time is $\tau_{\mathrm{dry}} \sim 20 \mathrm{~s}$, and the Péclet number is given by:

$$
P e=\frac{6 \pi \eta a}{k_{B} T} \cdot \frac{R^{2}}{\tau_{d r y}}
$$

where $\mathrm{R}$ is the droplet radius, a the radius of a zirconia particle, $\eta$ the viscosity of the suspending fluid and $\mathrm{k}_{\mathrm{B}} \mathrm{T}$ the thermal energy. For a millimetre sized droplet at $100^{\circ} \mathrm{C}$, Pe is close to $2 \times 10^{4}$.

In addition, sedimentation experiments were performed in polypropylene tubes (radius of $20 \mathrm{~mm}$ and volume of $25 \mathrm{~mL}$ ). Owing to the large density of zirconia (5.7), the gravitational length of the colloidal particles is smaller than $20 \mu \mathrm{m}$. Sedimentation is completed after two weeks and the height of the equilibrium sediment is measured, from which the sediment volume fractions of Table 2 have been deduced. At low ionic strength, the small values of the sediment volume fraction

Table 2: Sediment volumic fraction $\Phi$ sed and height measurements $\mathrm{H}$ of zirconia suspensions with different concentration in NaCl.

\begin{tabular}{c|cccccc}
$\mathrm{I}(\mathrm{mol} / \mathrm{L})$ & $3.1 \times 10^{-3}$ & $6.5 \times 10^{-3}$ & $1.2 \times 10^{-2}$ & $2.0 \times 10^{-2}$ & $3.7 \times 10^{-2}$ & $7.2 \times 10^{-2}$ \\
$\Phi_{\text {sed }}$ & 0.35 & 0.33 & 0.29 & 0.22 & 0.19 & 0.18 \\
$\mathrm{H}(\mathrm{mm})$ & 28 & 32 & 34 & 42 & 50 & 52
\end{tabular}

compared to the close packing volume fraction results from the high polydispersity of the particles. As the ionic strength increases, the decreasing values of $\Phi_{\text {sed }}$ indicate that particles are aggregated.

Diffusive Wave Spectroscopy (DWS) experiments were performed using a laboratory-made set-up. The coherent source is a laser operated at $\lambda=532 \mathrm{~nm}$ with an output power of $20 \mathrm{~mW}$. Suspensions samples for DWS measurements are prepared in cuvettes with a path length of $5 \mathrm{~mm}$. The diffused light is collected by an optical fibre placed in the transmission geometry to obtain an intensity autocorrelation function $g^{(2)}(t)$.

We recall that the function $\mathrm{g}^{(2)}(\mathrm{t})$ reveals the mean square displacement of the particles. The faster the decay, the faster the particles diffuse in the sample. No attempt was made to quantitatively measure the mean square displacement of the particles, because of the polydispersity of the various state of aggregation of the particles. The autocorrelation function $\mathrm{g}^{(2)}(\mathrm{t})$ of the various samples are gathered in Fig 3. $\mathrm{g}^{(2)}(\mathrm{t})$ decays with different kinetics, depending on the salt concentration revealing the aggregation of the particles. There is a clear frontier in the figure indicated by a black full line that separates the samples weakly aggregated that leads to buckling droplets with the one strongly aggregated that leads to spherical droplets. We will further comment that point in the next section. 


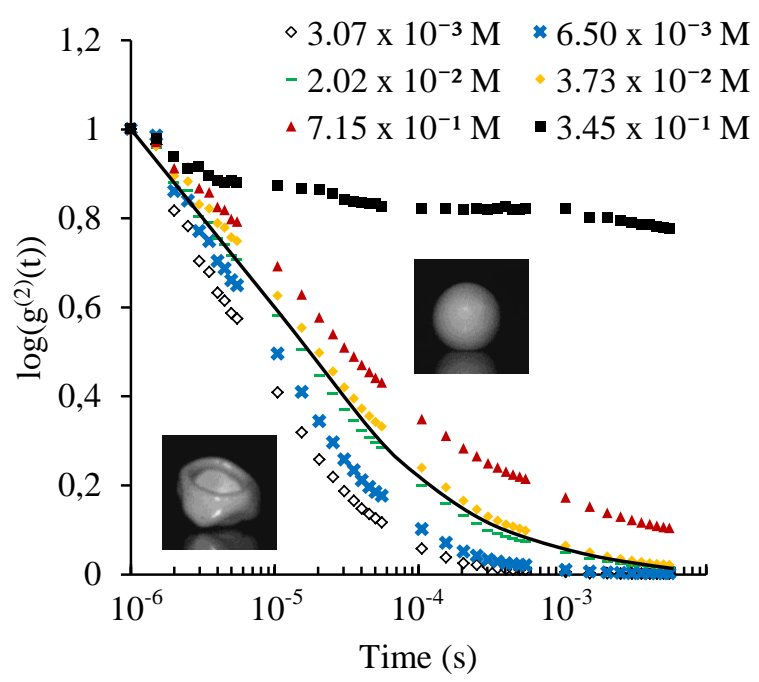

Fig. 3: Evolution of $\log \left(g^{(2)}(t)\right)$ normalized signals as a function of time for zirconia aqueous suspensions $50 \%$ wt with different ionic strength adjusted with $\mathrm{NaCl}$ additions. Continuous black line shows the critical function corresponding to the cup-sphere morphology transition.

\section{Results and discussion}

\section{Influence of the formulation on the morphology after drying.}

Drying of droplets of zirconia suspensions were performed for different initial salt contents. The results for increasing $\mathrm{NaCl}$ concentrations, corresponding to increasing ionic strengths, are shown in Fig. 1. At small ionic strengths buckling is observed, whereas for ionic strengths larger than $2.5 \times 10^{-2} \mathrm{~mol} / \mathrm{L}$, the droplets keep their spherical shape as they dry. For very large salt contents, droplets blow up during drying. A similar phenomenon was observed with concentrated solutions of salt (without colloids), suggesting formation of a solid crystallised shell that further implodes.

Similar experiments were performed with addition of polyacrylic acid at a fixed ionic strength. As pointed out above, PAA adsorbs on the particles surfaces, and modifies the zeta potential of the particles. The photographs of the droplets dried with different initial concentrations of PAA are shown in Fig. 2. In that case, two morphological transitions are observed as the PAA concentration increases: grains are spherical for polymer concentrations between 0.2 and $0.5 \% \mathrm{wt} / \mathrm{wt}$, corresponding to absolute values of the zeta potential smaller than $25 \mathrm{mV}$. For larger absolute values of the zeta potential, buckling is observed whatever the sign of the zeta potential.

We show in Fig. 4 the measurement of the droplet radius $\mathrm{R}$ as a function of time for a stable spherical shape (blue data) and when buckling occurs (red data). In both cases the radius decreases at the same - nearly constant rate but further reaches a constant value corresponding to the spherical dried state, whereas it is not defined any more when buckling occurs.

Since the Péclet number is large, during drying a concentrated shell forms at the interface with air. We denote as $h(t)$ the shell thickness and $r(t)$ the core radius as in Fig. 4. Conservation of the particle mass yields the relation:

$$
r^{3}=\frac{R^{3} \Phi_{\text {shell }}-R_{0}^{3} \Phi_{0}}{\Phi_{\text {shell }}-\Phi_{0}}=(R-h)^{3}
$$




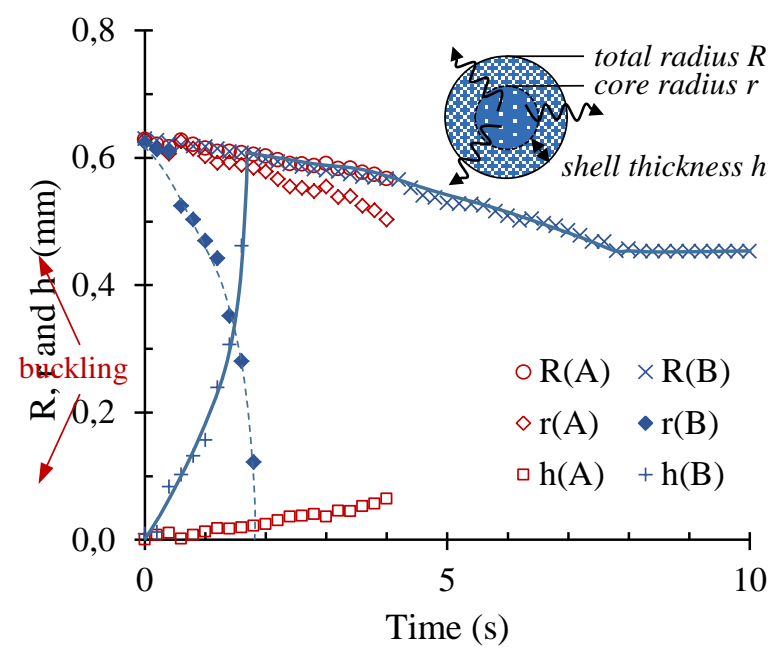

Fig. 4: Evolution of the total droplet radius $R$, core radius $r$ and shell thickness $h$ as functions of time. A (red dots) and B (blue d ots) corresponds to droplets of zirconia aqueous suspensions of two different ionic strengths (respectively $\mathrm{I}=3.1 \times 10^{-3} \mathrm{~mol} / \mathrm{L}$ and I $=3.7 \times 10^{-2} \mathrm{~mol} / \mathrm{L}$ ).

where $R_{0}$ is the initial droplet radius, $\Phi_{\text {shell }}$ and $\Phi_{0}$ the shell and initial volumic fractions of zirconia respectively. For each ionic strength, the value of the volume fraction $\Phi_{\text {shell }}$ can be estimated using the measured volume fractions of the sediment: $\Phi_{\text {shell }} \sim \Phi_{\text {sed }}$.

We will justify that point in the following by showing that the stresses exerted are of the same order of magnitude in both situations.

The computed variations of $h(t)$ and $r(t)$ are shown in Fig. 4 in the two considered cases: with and without buckling. As shown in the following, the Darcy stress resulting from the solvent flow through the shell can be inferred from the variations of $h(t)$.

\section{Critical aggregation pressure.}

Let us first focus on the experiments at varying ionic strength and given zeta potential (Fig. 1). For a more quantitative analysis, we have reported the buckling time as a function of the ionic strength of the colloidal suspension in Fig. 5. Buckling time is determined visually but drying is very reproducible so that buckling time is measured with a precision of $0.25 \mathrm{~s}$.

Three different regimes can be distinguished from the data of Fig. 5. First, as the ionic strength increases from its minimum value, the buckling time decreases. A minimum is reached, and as the ionic strength further increases, the buckling time then increases in a second regime. Finally, above $\mathrm{I}=5 \times 10^{-2} \mathrm{~mol} / \mathrm{L}$, no buckling is observed and the buckling time is therefore not defined in that third regime.

Those results can be understood as follows: buckling is preceded by the formation of a viscoelastic shell resulting from the colloidal aggregation induced by the solvent flow. As in Tsapis et al. ${ }^{12}$

, the first regime corresponds to colloidal interaction potentials with a high amplitude energy barrier, leading to initially well dispersed suspensions. During drying, water flows to the interface with air, which results in a Darcy stress across 


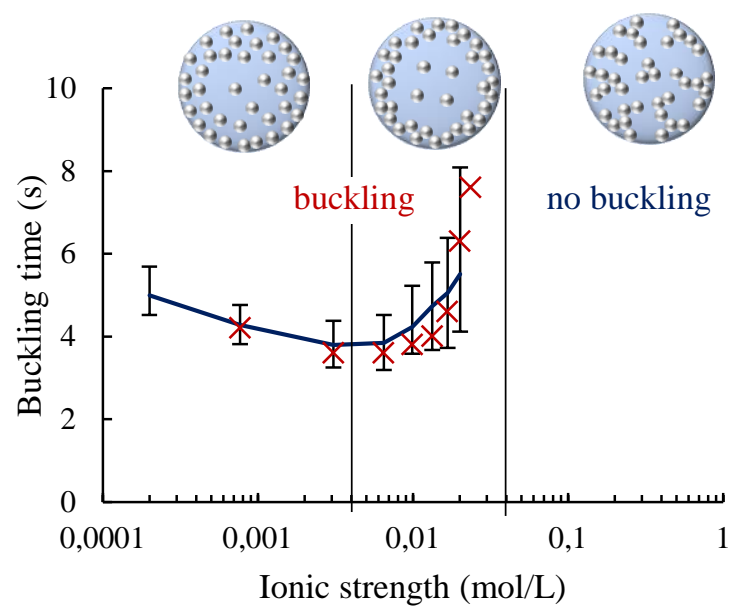

Fig. 5: Evolution of buckling time as a function of ionic strength. Red crosses correspond to experimental values whereas the blue curve corresponds to theoretical values computed from equation (12). Errors bars for experimental buckling times are too small to a ppear. Errors bars for calculated buckling times come from the measurement of zeta potential, input of equations (6) and (7).

the shell of thickness $\mathrm{h}(\mathrm{t})$. The Darcy stress is related to the rate of decrease of the droplet radius by:

$$
\sigma_{\text {Darcy }}(t) \sim \frac{\eta h(t)}{K} \frac{d R}{d t}
$$

where the permeability $\mathrm{K}$ of the shell can be estimated considering the shell is a packing of spheres of radius a at the volume fraction $\Phi_{\text {shell }}$ and using the Carman-Kozeny relation:

$$
K=\frac{a^{2}\left(1-\Phi_{\text {shell }}\right)^{3}}{45 \Phi_{\text {shell }}^{2}}
$$

As the droplet dries, the shell thickness, and therefore $\sigma_{\text {Darcy }}$ increases since the solvent velocity and permeability can be considered as constants. Buckling occurs when the Darcy pressure resulting from the solvent flow is large enough to induce aggregation of the colloidal particles. The DLVO potential for charged colloidal particles separated by a distance $\mathrm{x}<<$ a writes ${ }^{19}$ :

$$
W_{D L V O}(x)=\frac{64 \pi k_{B} \operatorname{Tan}^{2}}{\kappa^{2}} e^{-\kappa x}-\frac{A a}{12 x}
$$

where $\mathrm{n}$ is the number density of ions in the electrolyte, $\kappa$ the invert of the Debye length and $\Gamma$ is such that:

$$
\Gamma=\tanh \frac{e \zeta}{4 k_{B} T}
$$

The variations with the interparticle distance $\mathrm{x}$ of the DLVO potential is shown in Fig. 6. For small ionic strengths, it presents an energy barrier of amplitude much larger than $\mathrm{k}_{\mathrm{B}} \mathrm{T}$. The effect of the Darcy pressure can be accounted for by writing an interaction potential between particles as the sum of the DLVO potential and of the potential associated with the Darcy stress, such that:

$$
W(x)=W_{D L V O}(x)+W_{\text {Darcy }}=W_{D L V O}+\sigma_{D a r c y} \pi a^{2} x
$$




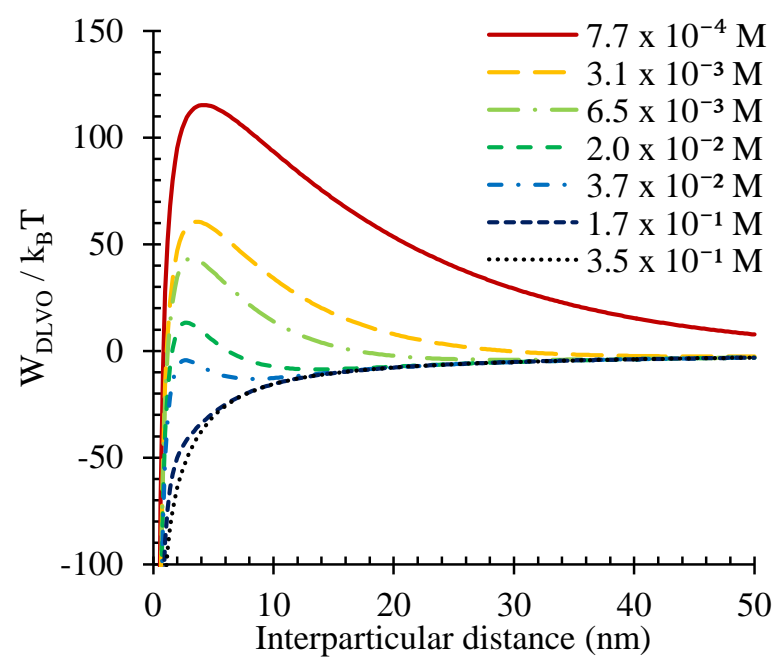

Fig. 6: DLVO interparticular potentials (normalized by $k_{B} T$ ) of zirconia aqueous suspensions with different ionic strengths.

Fig. 7 shows the variations of $\mathrm{W}(\mathrm{x})$ for given ionic strength and zeta potential, and different values of $\sigma_{\text {Darcy }}$. The height of the energy barrier is decreased and a minimum of potential appears. When the difference between the barrier height and the potential at its minimum reaches a value of a few $\mathrm{k}_{\mathrm{B}} \mathrm{T}$, particles can aggregate under the action of the Darcy stress, although their DLVO potential is strongly repulsive. We define the critical pressure $\mathrm{P}_{\mathrm{C}}$ as the Darcy pressure for which that difference is $10 \mathrm{k}_{\mathrm{B}} \mathrm{T}$ (see Fig. 7).

For larger ionic strengths, the height of the DLVO energy barrier decreases so that aggregation can spontaneously occurs within the time elapsed between the preparation of suspensions and the drying experiments. It is therefore a suspension of aggregates that is dried, instead of a suspension of well dispersed colloidal particles. As a result, the shell consists in a packing of aggregates, and its permeability is expected to be larger than for initially well dispersed suspensions. As in the classical Diffusion Limited Cluster Aggregation (DLCA) description, the structure of the aggregates is fractal, with a fractal dimension $D_{f}$ whose value can be estimated as 2.1 owing to the influence of gravity during the aggregation process ${ }^{20}$. The radius $\mathrm{R}_{\mathrm{A}}$ of an aggregate radius is given by ${ }^{21}$ :

$$
R_{A}=a\left(\frac{\Phi_{0}}{\Phi_{\text {shell }}}\right)^{\frac{1}{D_{f}-3}}
$$

The shell is therefore more permeable, which delays its buckling. The critical aggregation pressure can be calculated using the previously derived relations, and by replacing the particle radius a by the aggregate radius in equation 4 . The volume fraction can be estimated from the sedimentation experiments performed at different ionic strengths: $\Phi_{\text {shell }} \sim \Phi_{\text {sed }}$. 


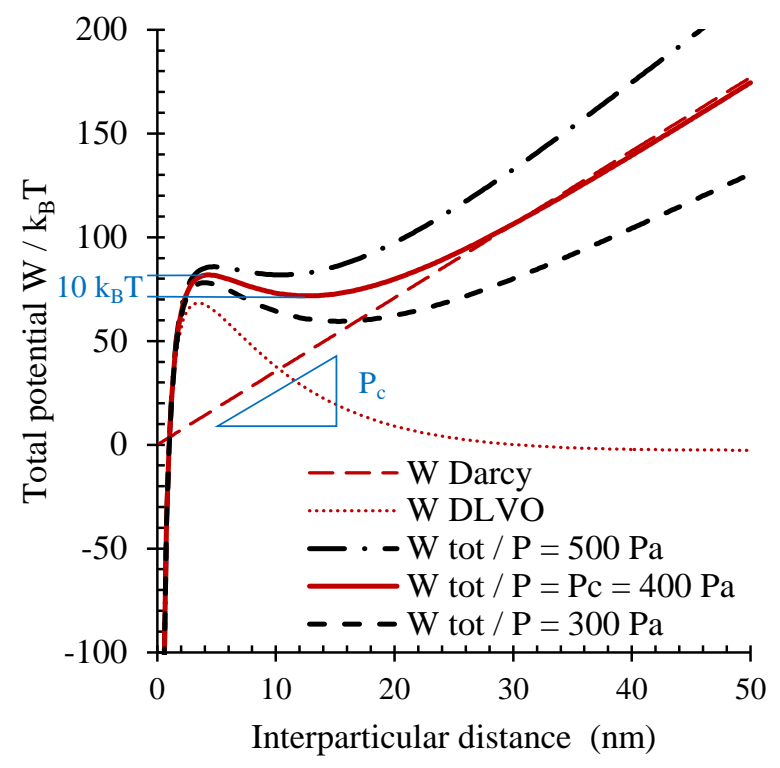

Fig. 7: Interparticular potentials (with DLVO potential and Darcy pressure) of zirconia aqueous suspensions with I $=6.50 \times 10^{-3} \mathrm{~mol} / \mathrm{L}$.

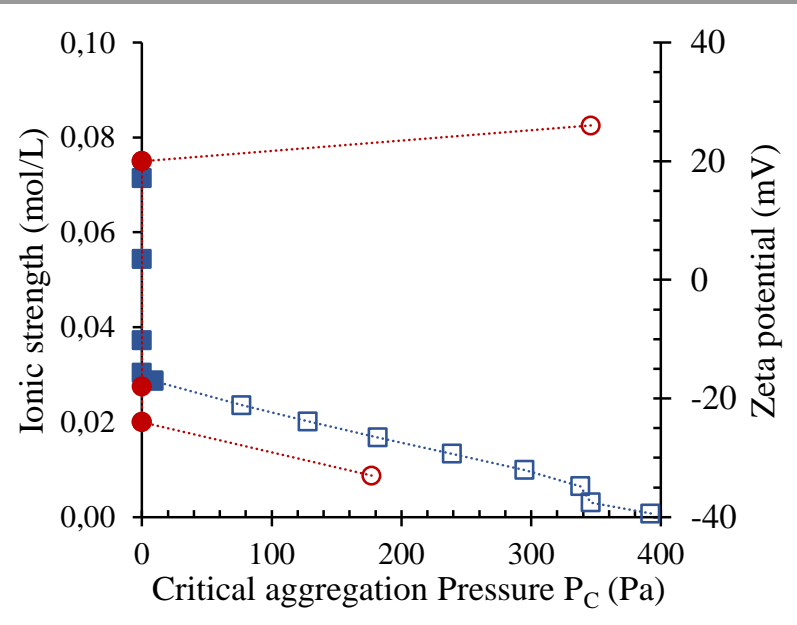

Fig. 8: Phase diagram of Leidenfrost grains for zirconia aqueous suspensions with different zeta potential (PAA formulations, right axis) or ionic strength ( $\mathrm{NaCl}$ formulations, left axis). Suspensions with a critical aggregation pressure below 50 Pa lead to spherical morphology. Filled and hollow symbols shows respectively spherical and cup shaped morphologies.

Figure 8 reports the critical aggregation pressure computed for each experiment of Fig. 1 and 2. When the ionic strength is increased (blue symbols), the critical aggregation pressure decreases to reach zero at large ionic strengths. Similarly, the data obtained at varying zeta potentials by adding PAA correspond to high aggregation pressure for large values of the zeta potential, and small pressures for small values of the zeta potential, leading to small amplitude of the DLVO energy barrier. In all cases, buckling is observed for critical aggregation pressures

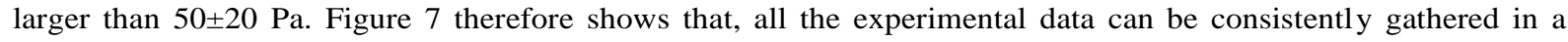
similar diagram using the critical aggregation pressure, whatever the way the interaction potential is modified. It thus confirms the relevance of the description we propose, based on an energy balance of the action of DLVO interactions and Darcy stress.

The assumption that the volume fraction in the shell can be estimated by the sediment volume fraction measured in the independent sediment experiments can be justified a posteriori. The stress exerted on a sediment of height $\mathrm{H}$ is given by:

$$
\sigma_{\text {Grav }} \sim g H \Phi_{\text {sed }} \Delta \rho
$$

where $\Delta \rho$ is the density difference of water and zirconia and $g$ the acceleration of gravity. According to the values given in Table 2, the value of $\sigma_{\text {Grav }}$ remains close to $400 \mathrm{~Pa}$, which is the same order of magnitude as $\sigma_{\text {Darcy }}$. 


\section{Buckling time.}

In addition, the buckling time can be quantitatively predicted from the same analysis. Assuming the shell thickness remains small compared to the radius droplet, a 1st order development of equation (3) yields the relation:

$$
h(t)=\frac{\Phi_{0}}{\Phi_{\text {shell }}-\Phi_{0}} \frac{d R}{d t} t
$$

Equation (11) is valid for constant drying rates, which is well verified experimentally (Fig. 1). Writing the condition for the onset of buckling, i.e. $\sigma_{\text {Darcy }}=\mathrm{P}_{\mathrm{c}}$, together with equations (4) and (9) gives the buckling time:

$$
t_{B}=\frac{P_{C} K}{\eta \frac{\Phi_{0}}{\Phi_{\text {shell }}-\Phi_{0}}\left(\frac{d R}{d t}\right)^{2}}
$$

For each ionic strength, the pressure $\mathrm{P}_{\mathrm{c}}$ has been computed as well as the permeability $\mathrm{K}$. The buckling time can therefore be computed following equation (12), and the corresponding values have been reported as a function of the ionic strength in Fig. 5. The non-monotonic behaviour of $t_{B}$ with ionic strength is fully predicted by equation (12): at small ionic strengths, the permeability $\mathrm{K}$ is constant and the aggregation pressure decreases with increasing ionic strength, leading to the observed decrease of the buckling time. The second regime corresponds to a permeability increasing faster with ionic strength than the aggregation pressure decreases, and thus to an increase of the buckling time. In addition, the experimental values of the buckling time correspond to the computed values within the uncertainty. A quantitative agreement is therefore found between experiments and the prediction of buckling from the colloidal interaction potential, which cannot be found with arguments based on stress balances as developed by Tsapis et al. ${ }^{17}$. We now turn to the cases in which no buckling is observed, i.e. when the spherical shape of the droplet remains stable.

\section{Spherical droplets}

We have shown in what precedes that, for small enough energy barriers of the DLVO potential, i.e. for small aggregation pressures, the droplets remain spherical during the whole drying process. In those situations, the colloidal suspension is initially partly aggregated and the shell is thus constituted by a packing of fractal aggregates with a large permeability $\left(\mathrm{K} \sim 2 \times 10^{-14} \mathrm{~m}^{2}\right.$ compared to $\mathrm{K} \sim 7 \times 10^{-16} \mathrm{~m}^{2}$ for well-dispersed suspensions). In consequence, the Darcy stress remains small and the droplets shrink isotropically without buckling. These results are consistent with the DWS measurements, which show that high ionic strengths implies aggregated suspensions. In particular, a gel does not need to be formed to induce spherical morphologies at the end of drying.

\section{Conclusions}

We have studied the drying of droplets of colloidal suspensions. The experimental conditions correspond to large Péclet numbers, leading to the formation of a shell concentrated in particles at the interface with air. As in previously reported experiments, the shell can buckle during drying. By tuning the interactions between colloidal particles, we show that the shape of the drying droplet is controlled by those interactions, and we provide a quantitative analysis of the phenomena. The full agreement of our predictions with the experiments opens the way for the controlled drying of colloidal suspensions.

\section{Acknowledgements}

This work is part of the thesis of E. Lintingre which is funded by the Agence Nationale de la Recherche Technologique and Saint-Gobain Innovative Materials. We thank T. Narita for his help in the DWS experiments.

\section{Notes and references}

Electronic Supplementary Information (ESI) available: [details of any supplementary information available should be included here]. See DOI: 10.1039/b000000x/

1. Lyonnard, S. et al. Sols. Langmuir 18, 10386-10397 (2002).

2. Pauchard, L. \& Allain, C.. Europhysics Letters (EPL) 62, 897-903 (2003).

3. Iskandar, F., Gradon, L. \& Okuyama, K. Journal of Colloid and Interface Science 265, 296-303 (2003).

4. Pauchard, L. \& Couder, Y. Europhysics Letters (EPL) 66, 667-673 (2004). 
5. Vehring, R., Foss, W. R. \& Lechuga-Ballesteros, D. Journal of Aerosol Science 38, 728-746 (2007).

6. Thybo, P., Hovgaard, L., Lindeløv, J. S., Brask, A. \& Andersen, S. K. Pharmaceutical Research 25, 1610-1620 (2008).

7. Roy, P., Bertrand, G. \& Coddet, C. Powder Technology 157, 20-26 (2005).

8. Rami, M.-L., Meireles, M., Cabane, B. \& Guizard, C. Journal of the American Ceramic Society 92, S50-S56 (2009).

9. Rogers, S., Fang, Y., Qi Lin, S. X., Selomulya, C. \& Dong Chen, X. Chemical Engineering Science 71, 75-84 (2012).

10. Bertrand, G., Roy, P., Filiatre, C. \& Coddet, C. Chemical Engineering Science 60, 95-102 (2005).

11. Lee, S. Y., Widiyastuti, W., Iskandar, F., Okuyama, K. \& Gradoń, L. Aerosol Science and Technology 43, 1184-1191 (2009).

12. Tsapis, N. et al. Physical Review Letters 94, (2005).

13. Bahadur, J. et al. Langmuir 28, 1914-1923 (2012).

14 Sen, D., Bahadur, J., Mazumder, S. \& Bhattacharya, S. Soft Matter 8, 10036 (2012).

15. Quemeneur, F., Quilliet, C., Faivre, M., Viallat, A. \& Pépin-Donat, B. Physical Review Letters 108, (2012).

16. Sen, D. et al. Soft Matter 8, 1955 (2012).

17. Boulogne, F., Giorgiutti-Dauphiné, F. \& Pauchard, L. Soft Matter 9, 750 (2013).

18. Biance, A.-L., Clanet, C. \& Quéré, D. Physics of Fluids 15, 1632 (2003).

19. Isrelachvili, J. Intermolecular \& Surface forces. (1991).

20. Senis, D., Gorre-Talini, L. \& Allain, C. The European Physical Journal E 4, 59-68 (2001).

21. Dubois, M. \& Cabane, B. Macromolecules 22, 2526-2533 (1989). 